\title{
Measurements and Simulations of Single-Event Upsets in a 28-nm FPGA
}

\author{
Markus Preston*, Per-Erik Tegnér \\ Stockholm University, Sweden \\ E-mail: markus.preston@fysik.su.se
}

Hans Calén, Tord Johansson, Karoly Makónyi, Pawel Marciniewski

Uppsala University, Sweden

\section{Myroslav Kavatsyuk, Peter Schakel}

KVI-CART, University of Groningen, the Netherlands

\begin{abstract}
Single-event upsets in the configuration memory of the 28-nm Xilinx Kintex-7 FPGA, used in the PANDA electromagnetic calorimeter, have been studied. Results from neutron and proton irradiations at energies up to $184 \mathrm{MeV}$ are presented and compared with previous experimental results. In order to gain information about the energy-dependence of the single-event upset cross section, a GEANT4-based Monte Carlo simulation of upset mechanisms in nanometric silicon volumes has been developed. The results from this model are shown to agree with the experimental data for both neutrons and protons. Knowledge about the energy dependence of the cross section and of the particle flux at the location of the front-end modules in PANDA enables better estimates of the mean time between failures in the electromagnetic calorimeter. At PANDA, a total neutron flux of $1 \cdot 10^{2} \mathrm{~cm}^{-2} \mathrm{~s}^{-1}$ at the location of the front-end modules is expected at the lowest antiproton beam momentum and a luminosity of $1 \cdot 10^{31} \mathrm{~cm}^{-2} \mathrm{~s}^{-1}$, leading to a predicted Mean Time Between Failures of $47 \pm 10$ hours per FPGA in the electromagnetic calorimeter.
\end{abstract}

Topical Workshop on Electronics for Particle Physics

11 - 14 September 2017

Santa Cruz, California

\footnotetext{
* Speaker.
} 


\section{Introduction}

The Facility for Antiproton and Ion Research (FAIR) is currently under construction in Darmstadt, Germany. One of the experiments at FAIR is PANDA (Anti-Proton Annihilation at Darmstadt), where antiprotons with momenta between $1.5 \mathrm{GeV} / c$ and $15 \mathrm{GeV} / c$ will interact in a hydrogen target.

The electromagnetic calorimeter (EMC) of PANDA will consist of approximately 16,000 lead tungstate $\left(\mathrm{PbWO}_{4}\right.$ or PWO) scintillating crystals, read out by avalanche photodiodes (APDs) and vacuum phototetrodes (VPTTs). The resulting signals will be processed by approximately 600 front-end digitiser modules, each featuring two 28-nm Xilinx Kintex-7 FPGAs. PANDA will be hardware-trigger-less, and these modules form the EMC software-based trigger.

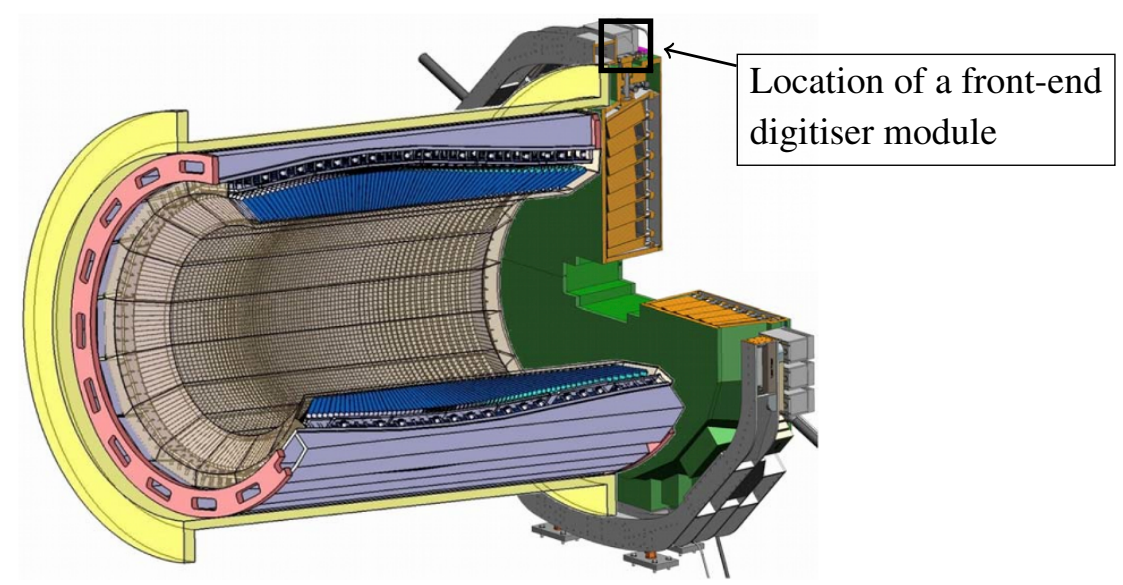

Figure 1: Schematic drawing of the barrel and forward-endcap parts of the PANDA electromagnetic calorimeter. The location of a front-end module in the forward endcap is shown. Figure modified from $[1]$.

Due to the high radiation exposure of the front-end modules, single-event upsets (SEUs) in the FPGA configuration memory are expected. These effects have been measured in the past $[2,3,4,7]$, but to estimate the rate of such errors in PANDA, information about the energy dependence of the SEU cross section is needed. This may be obtained through irradiations using known particle energies, combined with Monte Carlo simulations.

In the present work, one front-end module has been irradiated with neutrons and protons at different energies up to $184 \mathrm{MeV}$ and the SEU cross sections have been determined. A simulation of energy deposits in nanometric volumes in silicon has been developed, and reproduces the experimental results. The mean time between failures (MTBF) in the FPGA configuration memory during PANDA operation is predicted.

\section{Experimental results}

\subsection{Experiments}

During the irradiations, the front-end module was placed perpendicular to the particle beam, so that the particles hit the FPGA from the top. The module was powered on throughout the 
measurements, and the FPGA configuration memory was continuously monitored for SEUs by using the Xilinx Soft-Error Mitigation (SEM) Core, which also enabled automatic correction of single-bit SEUs and certain types of multi-bit SEUs.

\subsubsection{Neutron irradiation}

The module was irradiated with neutrons at The Svedberg Laboratory (TSL) in Uppsala, Sweden. The neutron beam had a continuous energy distribution from $0 \mathrm{MeV}$ to $180 \mathrm{MeV}$ [5], and the SEU cross section was determined to be $(7.4 \pm 0.6) \cdot 10^{-15} \mathrm{~cm}^{2} \mathrm{bit}^{-1}$. A comparison with results from previous experiments, made at TSL and at the Los Alamos Neutron Science Center (LANSCE), is shown in Fig. 2. Direct comparison with the LANSCE results is not possible due to the difference in the energy spectrum [5], but they are included for completeness. All of these results, including the one from this work, assume that only neutrons above $10 \mathrm{MeV}$ cause SEUs, in accordance with the JESD89A standard [6].

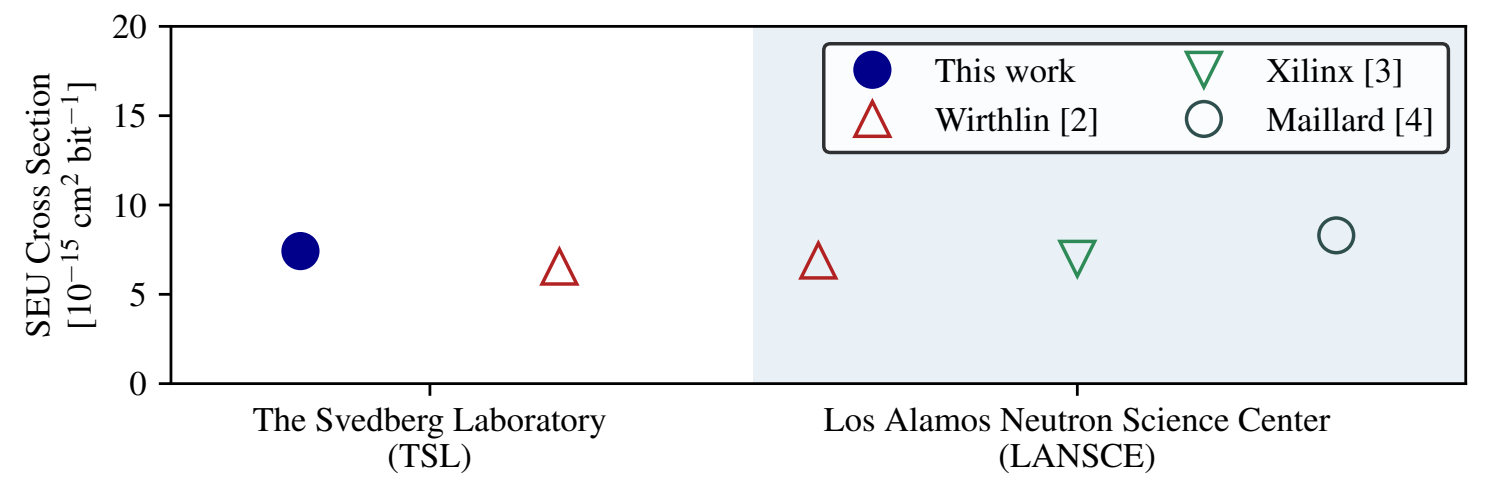

Figure 2: The measured cross section for neutron-induced SEUs in the Kintex-7 configuration memory, compared with results from previous experiments, performed both at the The Svedberg Laboratory and at the Los Alamos Neutron Science Center.

\subsubsection{Proton irradiation}

The module was irradiated with protons at KVI-CART in Groningen, the Netherlands. Measurements were performed at three beam energies: $80 \mathrm{MeV}, 100 \mathrm{MeV}$ and $184 \mathrm{MeV}$. The SEU cross sections at these three energies were determined to be $(7.9 \pm 0.9) \cdot 10^{-15} \mathrm{~cm}^{2} \mathrm{bit}^{-1},(6.7 \pm$ $0.5) \cdot 10^{-15} \mathrm{~cm}^{2}$ bit $^{-1}$ and $(5.7 \pm 0.6) \cdot 10^{-15} \mathrm{~cm}^{2}$ bit $^{-1}$, respectively. A comparison with results from previous experiments is shown in Fig. 3.

\section{Simulation results}

A GEANT4 model of a memory cell containing four cubic sensitive volumes (SVs) located within a silicon volume has been developed. Neutrons and protons with energies matching those in the experiments were directed into the cell (as shown in Fig. 4), and by using the GEANT4 MicroElec physics list [8] the interactions within the memory cell were modelled.

For each primary neutron or proton, the resulting energy deposits in the four SVs were determined, and if the deposited energy in any of the SVs was larger than a critical energy $E_{\text {crit }}$, the event 


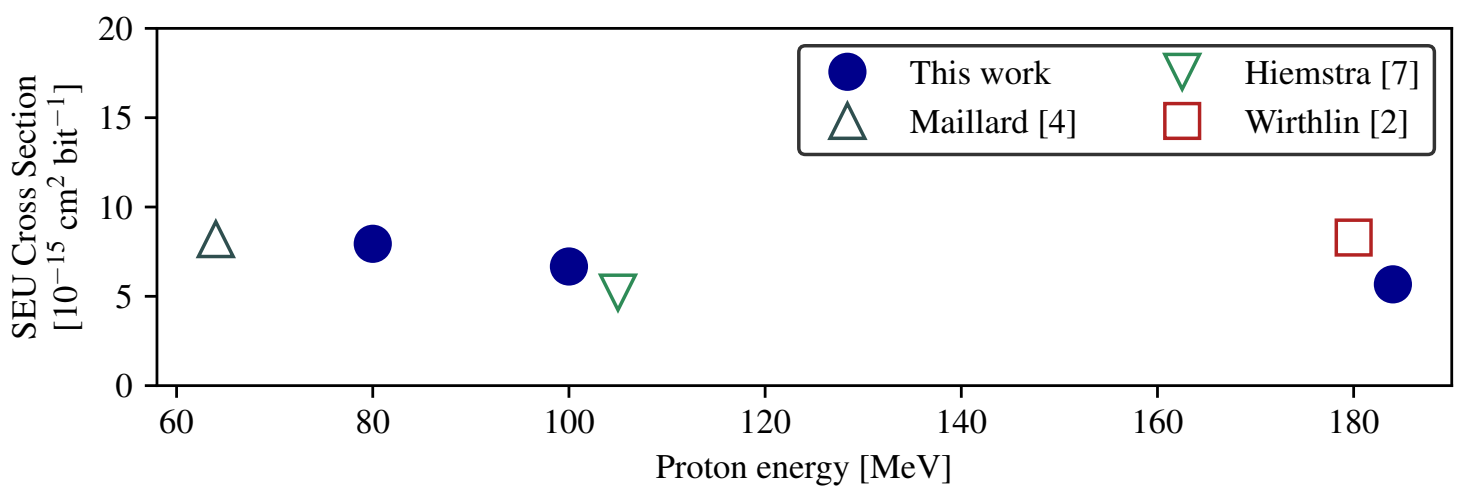

Figure 3: The measured cross section for proton-induced SEUs in the Kintex-7 configuration memory at proton energies of $80 \mathrm{MeV}, 100 \mathrm{MeV}$ and $184 \mathrm{MeV}$, compared with results from previous experiments.

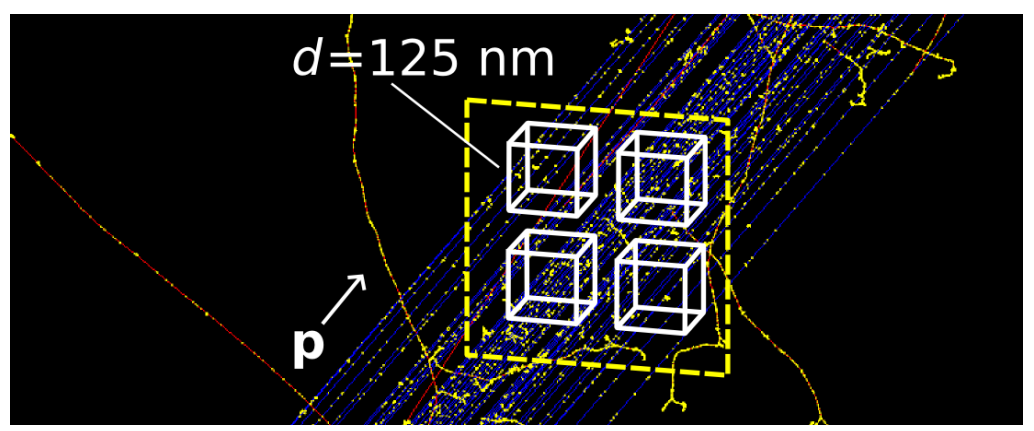

Figure 4: Visualisation of the GEANT4 simulation of $100 \mathrm{MeV}$ protons traversing a silicon block containing the four Sensitive Volumes (SVs), shown in white. The side d of the SV is $125 \mathrm{~nm}$. The incident proton beam covered an area of $400 \times 400 \mathrm{~nm}^{2}$, as shown by the dashed square.

was categorised as an SEU event. The critical energy and the SV size $d^{3}$ giving the best agreement with experiments were found through a global $\chi^{2}$ fit of the simulated SEU cross sections to the experimental SEU cross sections. With the resulting values $E_{\text {crit }}=4.6 \pm 0.2 \mathrm{keV}$ and $d=125 \pm 6$ $\mathrm{nm}$, both the experimental proton and neutron cross sections are reproduced as shown in Fig. 5.

The PandaRoot [9] framework was used to simulate $2 \cdot 10^{6} 1.5 \mathrm{GeV} / c \bar{p} p$ collisions in PANDA using a Dual Parton Model (DPM) event generator. Assuming the initial PANDA luminosity of $10^{31}$ $\mathrm{cm}^{-2} \mathrm{~s}^{-1}$, the maximum flux of neutrons at the location of the EMC digitisers was determined to be $1 \cdot 10^{2} \mathrm{~cm}^{-2} \mathrm{~s}^{-1}$. Based on the experimental and simulated cross sections, the MTBF for neutroninduced SEUs is $47 \pm 10$ hours per FPGA in the EMC under these conditions.

\section{Conclusions}

The measured cross sections agree with previous measurements, validating the experimental procedure. The simulation reproduces these results for both neutrons and protons, indicating a critical energy of $4.6 \pm 0.2 \mathrm{keV}$. With a critical energy this low, even low-energy neutrons and protons potentially cause SEUs - this will be studied in further experiments and simulations. Also, the effects of changing the size of the simulated beam and the depths of the SVs will be studied further, to determine to what extent the simulated cross sections depend on these parameters. 


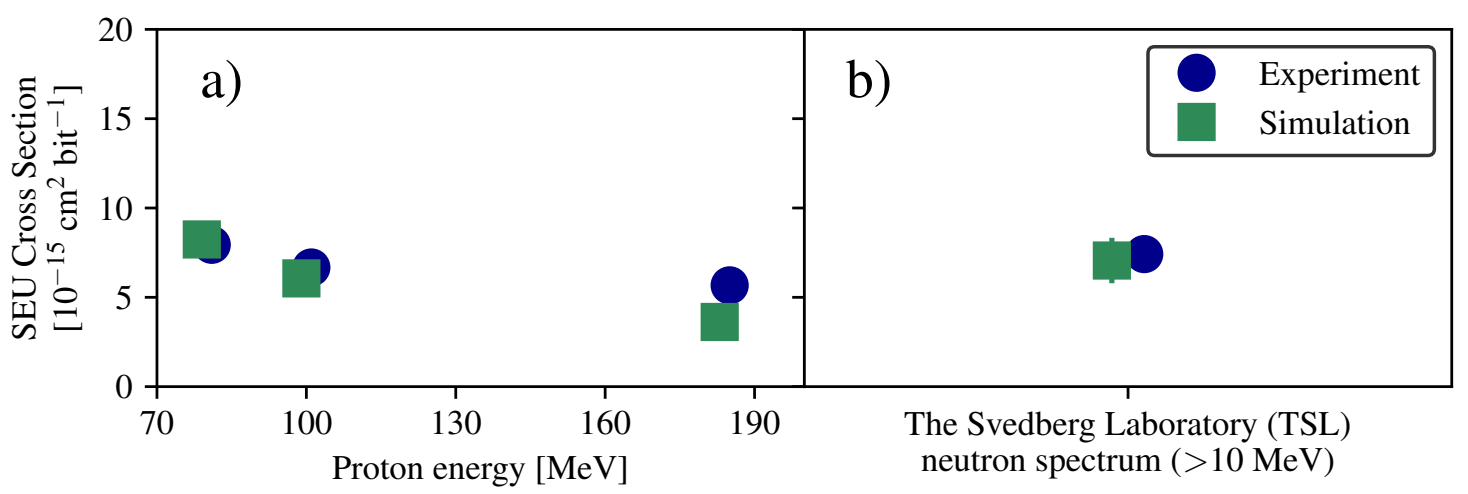

Figure 5: Comparison between the experimentally determined cross sections for a) proton-induced and b) neutron-induced SEUs and the results from the GEANT4 simulations for the corresponding particles and energies. The simulations were performed at the optimal SV size $d^{3}=125 \times 125 \times 125 \mathrm{~nm}^{3}$ and the optimal critical energy $E_{\text {crit }}=4.6 \mathrm{keV}$. The uncertainties in the simulated data are statistical.

Dependence of the MTBF on $\bar{p}$ momentum and luminosity will be studied further to determine the need for error mitigation in the FPGA when used in the EMC.

\section{References}

[1] M. Albrecht and the PANDA Collaboration, The Forward Endcap of the Electromagnetic Calorimeter for the PANDA Detector at FAIR, J. Phys: Conf. Ser. 587 (2015) 012050.

[2] M. J. Wirthlin, H. Takai and A. Harding, Soft error rate estimations of the Kintex-7 FPGA within the ATLAS Liquid Argon (LAr) Calorimeter, JINST 9 (2014) C01025.

[3] Xilinx, Device Reliability Report, First Half 2016, https://www.xilinx.com/support/documentation/user_guides/ug116.pdf [Accessed May 3, 2017].

[4] P. Maillard, M. Hart, J. Barton, P. Jain and J. Karp, Neutron, 64 MeV proton, thermal neutron and alpha single-event upset characterization of Xilinx 20nm UltraScale Kintex FPGA, IEEE Radiat. Effects Data Workshop (2015), 1-5.

[5] A. V. Prokofiev, J. Blomgren, M. Majerle, R. Nolte, S. Röttger, S. P. Platt, C. Xiao Xiao and A. N. Smirnov, Characterization of the ANITA Neutron Source for Accelerated SEE Testing at The Svedberg Laboratory, IEEE Radiat. Effects Data Workshop (2009), 166-173.

[6] JESD89A, Measurement and Reporting of Alpha Particle and Terrestrial Cosmic Ray-Induced Soft Errors in Semiconductor Devices, https://www.jedec.org/sites/default/files/docs/jesd89a.pdf [Accessed May 5, 2017].

[7] D. M. Hiemstra and V. Kirischian, Single Event Upset Characterization of the Kintex-7 Field Programmable Gate Array Using Proton Irradiation, IEEE Radiat. Effects Data Workshop (2014), $1-4$.

[8] M. Raine, M. Gaillardin and P. Paillet, Geant4 physics processes for silicon microdosimetry simulation: Improvements and extension of the energy-range validity up to $10 \mathrm{GeV} / n u c l e o n$, Nucl. Instrum. Meth. B 325 (2014) 97-100.

[9] S. Spataro and the PANDA Collaboration, The PandaRoot framework for simulation, reconstruction and analysis, J. Phys: Conf. Ser. 331 (2011) 032031. 